Article

\title{
Total, Added, and Free Sugar Consumption and Adherence to Guidelines in Switzerland: Results from the First National Nutrition Survey menuCH
}

\author{
Angeline Chatelan ${ }^{1, *(\mathbb{D}}$, Pierre Gaillard ${ }^{1}$, Maaike Kruseman ${ }^{2}\left(\mathbb{D}\right.$ and Amelie Keller ${ }^{3}$ \\ 1 Center of Primary Care and Public Health (Unisanté), University of Lausanne, Route de la Corniche 10, \\ 1010 Lausanne, Switzerland; pierre.gaillard@etu.hesge.ch \\ 2 School of Health Sciences, HES-SO University of Applied Sciences and Arts Western Switzerland, \\ Rue des Caroubiers 25, 1227 Carouge, Geneva, Switzerland; maaike.kruseman@hesge.ch \\ 3 Research Unit for Dietary Studies at the Parker Institute, Bispebjerg og Frederiksberg Hospital, The Capital \\ Region, Nordre Fasanvej 57, 2000 Frederiksberg, Denmark; amelie.cleo.keller@regionh.dk \\ * Correspondence: Angeline.Chatelan@chuv.ch or a.chatelan@bluemail.ch; Tel.: +41-314-8238
}

Received: 28 March 2019; Accepted: 16 May 2019; Published: 19 May 2019

check for updates

\begin{abstract}
The World Health Organization (WHO) recommends reducing free sugars to less than $10 \%$ of total energy intake (TEI) due to their potential implications in weight gain and dental caries. Our objectives were to (1) estimate the intake of total, added, and free sugars, (2) define the main sugar sources, and (3) evaluate the adherence to sugar guidelines. The first national nutrition survey 2014-2015 included non-institutional adults aged 18-75 years. Diet was assessed with two non-consecutive 24-hour dietary recalls in 2057 participants. Added and free sugar content was systematically estimated by two dietitians using available information from the manufacturer and/or standard recipe/composition. Usual daily intake distributions were modeled and weighted for sampling design, non-response, weekdays, and seasons. Total, added, and free sugar intake was respectively $107 \mathrm{~g}( \pm 44), 53 \mathrm{~g}( \pm 36)$, and $65 \mathrm{~g}( \pm 40)$, representing 19\%, 9\%, and 11\% of TEI. Sugar consumption was higher among younger adults and lower among people living in the Italian-speaking region. The three main food sources of free sugars were: (1) sweet products (47\% of total free sugars), in particular sweet spreads (15\%) and cakes/cookies (11\%); (2) beverages $(29 \%)$, mainly fruit and vegetable juices $(13 \%)$, and sugar-sweetened beverages $(12 \%$, but $20 \%$ in younger adults); and (3) dairy products (9\%), with yogurt accounting for $6 \%$. Respectively, $44 \%$ of women and $45 \%$ of men had free sugar intake below $10 \%$ of TEI. Of people aged between 18-29, 30-64, and 65-75 years, 36\%, $45 \%$, and $53 \%$ had free sugar intake below $10 \%$ of TEI, respectively. The prevalence of Swiss people with free sugar intake that was $<5 \%$ of the TEI was $8 \%$. Adherence to the WHO recommendations guidelines was generally low in Switzerland, particularly among young adults, and in line with other high-income countries.
\end{abstract}

Keywords: sugar intake; dietary survey

\section{Introduction}

Several systematic reviews and meta-analyses have concluded that an increased intake of sugar and sugar-sweetened beverages (SSBs) is directly associated with weight gain, overweight, and obesity [1-3]. The intake of dietary sugars has also a deleterious effect on oral health, and is the most important risk factor for dental caries [4,5]. In addition to dental caries and body weight, excessive sugar consumption has been associated with an increased risk of developing several chronic diseases, such as type 2 diabetes [6], cardiovascular diseases [7-9], and some cancers [10-12], as well as non-alcoholic fatty liver disease [13]. Emerging research suggests that a diet high in sugars may increase the risk of 
developing dementia such as Alzheimer disease [14,15]. In this context, the World Health Organization (WHO) recommends a reduced intake of free sugars throughout the life course with a reduction of free sugars intake to less than $10 \%$ of total energy intake (TEI, strong recommendation) and preferably below $5 \%$ of TEI (conditional recommendation) in both adults and children [16]. In Switzerland-a wealthy country with three main linguistic regions, one of the highest life expectancies [17], and the lowest prevalence of obesity in high-income countries [18] — the maximum daily intake for free sugars is set at $50 \mathrm{~g}$, corresponding to $10 \%$ of a TEI equivalent to $2000 \mathrm{kcal}$ [19].

In Europe, sugar consumption currently contributes between $7-11 \%$ and $11-17 \%$ of TEI in adults and children, respectively [20]. Worldwide, data suggest that added sugar intake rises starting from one year of age, and is highest among school-age children and adolescents compared to adults [21]. Sweet products (i.e., cakes, biscuits, pastries, confectionary, jam/honey, ice cream, table sugar), beverages (i.e., SSBs and fruit nectars, excluding fruit juices) and dairy products (i.e., yoghurts, milk-based desserts) contribute the most to added sugar intake in both adults and children [20,22].

Around the world, a variety of public health policies have been designed to limit the intake of added or free sugar, such as food labeling (i.e., Nutri-score) [23], food reformulation [24], a reduction of portion sizes [25], and the taxation of SSBs [26]. In Switzerland, several policy proposals or public health interventions are being considered [27]. However, in order for these policies to be evaluated in the future, reliable data about the current sugar intake of the population is needed. These data are also necessary for monitoring purposes and to investigate who are the most at risk of sugar overconsumption. Yet, in Switzerland, the population's sugar consumption has not previously been reported. Hence, the aims of this study were (1) to estimate the intake of total, added, and free sugars, (2) to define the main sugar sources at the food group level, and (3) to evaluate the adherence to sugar guidelines in the Swiss adult population using data from the first national nutrition survey, menuCH.

\section{Methods}

\subsection{Study Design and Population}

The cross-sectional Swiss National Nutrition Survey, menuCH, was conducted among non-institutionalized residents aged 18-75 years old [28]. Participants were recruited from the national sampling frame for person and household surveys [29] using a stratified random sample design. The survey population was intended to be representative of the Swiss population in terms of age and place of residency (all seven major areas of Switzerland) [28]. A total of 5496 eligible people reachable by phone were invited, of which $2086(38 \%)$ responded [28]. Participants and non-participants had similar age and marital status, but participants were more frequently women and Swiss nationals [28]. Data were collected from January 2014 to February 2015. Further information about menuCH is available here: https://menuch.iumsp.ch. The study was registered in the trial registry (identification number: ISRCTN16778734) and conducted according to the guidelines laid down in the Declaration of Helsinki. Each participant signed a written informed consent.

\subsection{Dietary Assessment}

Trained dietitians conducted two non-consecutive 24-h recalls (24HDR, first: face-to-face and second: by phone, two to six weeks later). They used the computer-directed interview program GloboDiet ${ }^{\circledR}$, which was previously known as EPIC-Soft ${ }^{\circledR}$ (International Agency for Research on Cancer (IARC), Lyon, France) [30,31] following standardized steps for 24HDR: (1) general participant information (e.g., special diet, special day); (2) quick list of food consumption occasions and items; and (3) detailed description and quantification of all consumed foods and beverages, including conservation and preparation methods, sugar content/addition, and portion size. To support survey participants in quantifying consumed amounts, dietitians used a book with 119 series of six graduated portion-size pictures [32]. 24HDR were spread over all weekdays and seasons. According to the description reported by the dietitians, each food item was linked to the best match in an extended research version 
of the 2015 Swiss Food Composition Database [33] using FoodCASE (Premotec GmbH, Winterthur, Switzerland) [34]. We classified all foods and beverages into 30 food groups, based on their original classification from GloboDiet ${ }^{\circledR}$ (18 groups and 85 subgroups) and on their nutritional similarity regarding sugar content.

\subsection{Total, Added, and Free Sugars: Definitions, Calculations, and Recommendations}

Supplementary Figures S1 and S2 provide an overview of the total, added, and free sugar definitions. Total sugars correspond to all monosaccharides and disaccharides present in food and derived from any source [16,35]. The Swiss Food Composition Database [33] was used to assign total sugars. This table includes 1000 generic/unbranded food items and 9600 branded items (data provided by industry) [33]. Information on total sugar content was missing in $1 \%$ of all consumed foods. If these foods were known to be significant sources of total sugars and were reported more than 15 times by survey participants, we assigned them a total sugar value that was based on similar products included in the Swiss Food Composition Database, manufacturer websites, or the online French Food Composition Table (Ciqual) [36]. Added sugars were defined as all sugars that are added during food processing and preparation (e.g., white and brown sugar/sucrose, glucose, high-fructose corn syrup, dextrose, fructose, honey, invert sugar, and lactose) [35,37]. Added sugars did not include lactose in dairy products nor sugars naturally occurring in fruit juices and concentrates, and unprocessed foods, such as fruit, vegetables, legumes, potatoes, fish, meat, poultry, and eggs [35,38]. WHO defines free sugars as "all sugars that are added during food manufacturing and preparation as well as sugars that are naturally present in honey, syrups, fruit juices and fruit concentrates" [16]. In 2018, Swan et al. [39] suggested a stricter definition of free sugars for the United Kingdom. In addition to the WHO definition, the British authors included all sugars contained in pureed fruit, fruit canned in syrup, and all types of alcoholic and non-alcoholic drinks [39]. We decided to comply with Swan's definition of free sugars. Supplementary Table S1 gives an overview of the decisions made and the number of food items assigned to the 30 food groups and their subgroups.

Two dietitians systematically and independently assigned the added sugar content for each food consumed by survey participants. Their estimations were based on standard recipes, information on packaging, or manufacturer websites $[37,38]$. When information was limited, added sugar content was estimated as the amount of total sugars minus the amount of natural occurring sugars estimated from a similar unsweetened food item (e.g., for apple puree and yogurts, see Supplementary Table S1) [37,38]. Free sugars were equal to added sugars, except for the sugars naturally present in all alcoholic and non-alcoholic drinks (including fruit juices and concentrates), pureed fruit, and fruit canned in syrup (see above and Supplementary Table S1).

\subsection{Covariates}

Sociodemographic information was collected by questionnaire: i.e., sex, age, education (highest degree $=$ university degree: yes/no), nationality (Swiss/non-Swiss). Language region was assigned according to home address. The German-speaking region included the cantons of Aargau, Basel-Land, Basel-Stadt, Bern, Lucerne, St. Gallen, Zurich; the French-speaking region included Geneva, Jura, Neuchatel, and Vaud; and the Italian-speaking region included Ticino.

\subsection{Statistical Analyses}

The usual daily consumption of total, added, and free sugars as well as total carbohydrate (CHO) and TEI was modeled out of the two 24HDR using the Multiple Source Method (MSM, https://nugo.dife.de/msm) [40]. The latter has been developed to assess the long-term average intake from short-term measurements, accounting for day-to-day variations (within-person variation). In MSM, we assumed that the survey participants were all potential consumers of total, added, and free sugars. The intakes of total, added, and free sugars (in g/day) and the percentages of TEI, CHO, or total sugars are presented by sex, age group, language region, educational level, and nationality. The 
proportions of persons adhering to international and national recommendations were stratified by the same categories. The main food sources of total added and free sugars are presented within 30 food groups. The average contribution percentage of each group was estimated using two-day mean intake. All the descriptive results were weighted for small deviations in sociodemographic characteristics (age, sex, marital status, place of residency, nationality, and household size), weekday, and seasonality [28]. The weighing strategy tends to limit bias due to the sampling design and non-response; it intends to provide results that are more representative of the Swiss population and of any day of the year. The weighing factors were computed in an iterative process using the 2014 sampling frame as the reference population $[28,29]$. We carried out all the statistical analyses using STATA version 14 (Stata Corp., College Station, TX, USA).

\section{Results}

Table 1 details the total, added, and free sugar consumption in the Swiss adult population. The mean daily total sugar intake was $107 \mathrm{~g}( \pm 44)$, contributing $19 \%$ of TEI. The mean daily added sugar intake was $53 \mathrm{~g}( \pm 36)$, contributing to $9 \%$ of TEI, and the mean daily free sugar intake was $65 \mathrm{~g}( \pm 40)$, representing $11 \%$ of TEI. Added and free sugar intakes contributed to $48 \%$ and $59 \%$ of total sugar intake, respectively (Table 1). The proportion of carbohydrate intake consumed as sugars was higher among women than men ( $49 \%$ versus $43 \%$ of $\mathrm{CHO}$ ), whereas a larger proportion of total sugar was consumed as free sugars among men (62\% of total sugars, versus $55 \%$ in women). Young adults aged 18 to 29 years had a higher mean $( \pm \mathrm{SD})$ consumption of total $(113 \mathrm{~g} \pm 47)$, added $(54 \mathrm{~g} \pm 36)$, and free sugar $(66 \mathrm{~g} \pm 40)$ compared to older adults aged 65 to 75 years (total sugar: $99 \mathrm{~g} \pm 38$; added sugar: $42 \mathrm{~g} \pm 25$; free sugar: $53 \mathrm{~g} \pm 28$ ) (Table 1). Individuals living in the Italian-speaking region had a lower mean intake of free sugar $(51 \mathrm{~g} \pm 38)$ compared to the French-speaking $(64 \mathrm{~g} \pm 38)$ and German-speaking regions $(66 \mathrm{~g} \pm 40)$. Intakes of total, added, and free sugar were similar between persons with or without a university degree as well as individuals with or without Swiss nationality (Table 1).

Table 2 shows the main food group sources of total, added, and free sugars. The three main sources of total sugars were (1) sweet products $(28 \%),(2)$ fruit $(21 \%)$, and (3) beverages (20\%). Main sources of added sugars were (1) sweet products (55\%), (2) beverages $(17 \%)$, and (3) dairy products $(10 \%)$. Main sources of free sugars were (1) sweet products $(47 \%$, in particular sweet spreads $(15 \%)$ and cakes/cookies $(11 \%))$, (2) beverages (29\%, mainly fruit and vegetable juices $(13 \%)$ and soft drinks/SSB $(12 \%))$, and (3) dairy products (9\%, with yogurt accounting for $6 \%$ ). These same three food groups were the largest contributors of free sugar, independently of age and gender (Tables 2 and 3). However, among younger adults, a bigger proportion of free sugars came from beverages, mainly SSBs (20\%), compared to older adults, whose main source of free sugar came from sweet products.

Table 4 provides information about adherence to WHO and national recommendations. Respectively, $44 \%$ of women and $45 \%$ of men had a free sugar intake below $10 \%$ of TEI. Among people aged 18 to 29,30 to 64 , and 65 to 75 years, $36 \%, 45 \%$, and $53 \%$ had free sugar intake below $10 \%$ of TEI $(p=0.001)$. A bigger proportion of individuals from the Italian-speaking region had a free sugar intake below $10 \%$ of TEI compared to the French-speaking and German-speaking regions, which were $62 \%, 42 \%$, and $44 \%$, respectively ( $p=0.002$ ). The prevalence of the population with a free sugar intake below $5 \%$ of TEI was $8 \%$ (Table 4); this figure was slightly higher in the Italian-speaking region, with a proportion of $13 \%$. 
Table 1. Weighted habitual intakes of total, added, and free sugars. TEI: total energy intake.

\begin{tabular}{|c|c|c|c|c|c|c|c|c|c|c|c|c|c|c|c|c|c|c|c|c|c|c|c|}
\hline & \multirow[b]{2}{*}{$\begin{array}{l}\mathrm{N} \\
(\%)\end{array}$} & \multirow[b]{2}{*}{$\begin{array}{l}\text { Weighted } \\
\mathbf{N}(\%)\end{array}$} & \multicolumn{7}{|c|}{ Total Sugars } & \multicolumn{7}{|c|}{ Added Sugars } & \multicolumn{7}{|c|}{ Free Sugar } \\
\hline & & & $\begin{array}{c}\text { Mean } \\
\text { in g }\end{array}$ & SD & $\begin{array}{c}\text { Median } \\
\text { in } \mathrm{g}\end{array}$ & P25 & P75 & $\begin{array}{l}\% \mathrm{TEI} \\
\text { (mean) }\end{array}$ & $\begin{array}{l}\text { \% Total } \\
\text { CHO } \\
\text { (mean) }\end{array}$ & $\begin{array}{l}\text { Mean } \\
\text { in g }\end{array}$ & $\mathrm{SD}$ & $\begin{array}{l}\text { Median } \\
\text { in } \mathrm{g}\end{array}$ & P25 & P75 & $\begin{array}{l}\% \text { TEI } \\
\text { (mean) }\end{array}$ & $\begin{array}{l}\% \text { Total } \\
\text { Sugars } \\
\text { (mean) }\end{array}$ & $\begin{array}{l}\text { Mean } \\
\text { in } g\end{array}$ & SD & $\begin{array}{l}\text { Median } \\
\text { in } \mathrm{g}\end{array}$ & P25 & P75 & $\begin{array}{l}\% \text { TEI } \\
\text { (mean) }\end{array}$ & $\begin{array}{l}\% \text { Total } \\
\text { Sugars } \\
\text { (mean) }\end{array}$ \\
\hline $\begin{array}{l}\text { All } \\
\text { Sex }\end{array}$ & $\begin{array}{l}2057 \\
(100) \\
933\end{array}$ & $\begin{array}{l}4,627,878 \\
(100) \\
2,305,141\end{array}$ & 107 & 44 & 100 & 76 & 130 & 19.3 & 46.0 & 53 & 36 & 44 & 29 & 66 & 9.3 & 48.0 & 65 & 40 & 56 & 38 & 83 & 11.5 & 58.6 \\
\hline Men & $\begin{array}{l}(45) \\
1124\end{array}$ & $\begin{array}{c}(50) \\
2,322,737\end{array}$ & 114 & 49 & 108 & 80 & 142 & 18.1 & 43.4 & 61 & 41 & 50 & 33 & 79 & 9.4 & 51.2 & 73 & 44 & 65 & 43 & 96 & 11.4 & 62.0 \\
\hline Women & (55) & (50) & 99 & 37 & 94 & 73 & 119 & 20.5 & 48.5 & 46 & 28 & 39 & 27 & 56 & 9.3 & 44.8 & 57 & 32 & 50 & 34 & 69 & 11.5 & 55.3 \\
\hline $\begin{array}{l}\text { Age } \\
18-29 y\end{array}$ & $\begin{array}{l}400 \\
(19) \\
1319\end{array}$ & $\begin{array}{c}870,489 \text { (19) } \\
3,108,362\end{array}$ & 113 & 47 & 109 & 79 & 137 & 19.6 & 44.0 & 63 & 41 & 53 & 33 & 81 & 10.5 & 52.7 & 75 & 45 & 67 & 42 & 97 & 12.7 & 63.5 \\
\hline $30-64 \mathrm{y}$ & $\begin{array}{l}(64) \\
338 \\
(16)\end{array}$ & 649,026 (14) & 107 & 38 & 100 & 77 & 130 & 19.5 & 45.9 & 53 & 36 & 37 & 26 & 52 & 8.1 & 48.0 & 65 & 28 & 56 & 38 & 82 & 11.4 & 58.6 \\
\hline $\begin{array}{l}\text { Linguistic region } \\
\text { German-speaking }\end{array}$ & $\begin{array}{l}1341 \\
(65) \\
502\end{array}$ & $\begin{array}{c}3,183,216 \\
(69) \\
1,187,738\end{array}$ & 110 & 44 & 104 & 78 & 134 & 19.5 & 46.2 & 54 & 36 & 46 & 30 & 68 & 9.4 & 47.6 & 66 & 40 & 58 & 39 & 87 & 11.6 & 58.2 \\
\hline $\begin{array}{l}\text { French-speaking } \\
\text { Italian-speaking }\end{array}$ & $\begin{array}{l}(24) \\
214 \\
(10)\end{array}$ & $256,925(6)$ & 103 & 43 & $\begin{array}{l}95 \\
84\end{array}$ & 73 & 121 & $\begin{array}{l}19.1 \\
17.3\end{array}$ & 46.1 & 52 & 35 & $\begin{array}{l}44 \\
33\end{array}$ & 29 & 64 & $\begin{array}{l}9.4 \\
8.1\end{array}$ & $\begin{array}{l}49.3 \\
46.9\end{array}$ & 64 & 38 & 56 & 36 & 79 & $\begin{array}{l}11.6 \\
9.7\end{array}$ & 60.2 \\
\hline $\begin{array}{l}\text { Education (highest } \\
\text { degree) }^{1} \\
\text { No university degree }\end{array}$ & $\begin{array}{l}1057 \\
(51) \\
997 \\
(48)\end{array}$ & $\begin{array}{c}2,210,585 \\
(48) \\
2,405,018 \\
(52)\end{array}$ & 105 & 46 & 97 & 72 & 127 & 19.3 & 46.1 & 54 & 38 & 44 & 28 & 66 & 9.6 & 48.9 & 65 & 42 & 56 & 36 & 82 & 11.7 & 59.5 \\
\hline $\begin{array}{l}\text { University degree } \\
\text { Nationality }{ }^{1} \\
\text { Swiss }\end{array}$ & $\begin{array}{l}1789 \\
(87)\end{array}$ & $\begin{array}{c}(52) \\
3,470,404 \\
(75)\end{array}$ & 107 & 43 & 101 & 77 & 131 & 19.5 & 46.2 & 53 & 34 & 45 & 30 & 68 & 9.4 & 47.9 & 65 & 38 & 58 & 38 & 84 & 11.5 & 58.7 \\
\hline Non-Swiss & $\begin{array}{l}265 \\
(13)\end{array}$ & $\begin{array}{c}1,145,199 \\
(25)\end{array}$ & 105 & 45 & 97 & 73 & 126 & 18.8 & 45.1 & 53 & 40 & 41 & 28 & 63 & 9.2 & 48.0 & 64 & 43 & 53 & 36 & 79 & 11.2 & 58.1 \\
\hline
\end{tabular}

${ }^{1}$ Three survey participants did not answer this question. 
Table 2. Mean contribution by food group for total, added, and free sugars in the entire population and by sex.

\begin{tabular}{|c|c|c|c|c|c|c|c|c|c|}
\hline \multirow[b]{2}{*}{ Food group } & \multicolumn{3}{|c|}{ All $(n=2057$, Weighted $n=4,627,878)$} & \multicolumn{3}{|c|}{ Men $(n=933$, Weighted $n=2,305,141)$} & \multicolumn{3}{|c|}{ Women $(n=1,124$, Weighted $n=2,322,737)$} \\
\hline & $\begin{array}{l}\text { Total Sugars } \\
\quad(\%)\end{array}$ & $\begin{array}{c}\text { Added } \\
\text { Sugars (\%) }\end{array}$ & $\begin{array}{l}\text { Free Sugars } \\
(\%)\end{array}$ & $\begin{array}{l}\text { Total Sugars } \\
\quad(\%)\end{array}$ & $\begin{array}{c}\text { Added } \\
\text { Sugars (\%) }\end{array}$ & $\begin{array}{l}\text { Free sugars } \\
\quad(\%)\end{array}$ & $\begin{array}{l}\text { Total Sugars } \\
(\%)\end{array}$ & $\begin{array}{c}\text { Added } \\
\text { Sugars (\%) }\end{array}$ & $\begin{array}{l}\text { Free Sugars } \\
(\%)\end{array}$ \\
\hline Tubercles and potato products & 0.5 & 0.4 & 0.3 & 0.6 & 0.4 & 0.2 & 0.5 & 0.4 & 0.3 \\
\hline Vegetables & 6.6 & 0.9 & 0.6 & 6.1 & 0.9 & 0.6 & 7.2 & 0.8 & 0.6 \\
\hline Fruit & 20.7 & 0.2 & 1.3 & 17.5 & 0.2 & 1.0 & 23.8 & 0.2 & 1.6 \\
\hline Nuts, seeds, and olives & 0.3 & 0.0 & 0.0 & 0.2 & 0.0 & 0.0 & 0.4 & 0.0 & 0.0 \\
\hline Dairy products & 13.0 & 10.0 & 8.6 & 12.4 & 9.5 & 8.2 & 13.6 & 10.5 & 8.9 \\
\hline Milk & 5.0 & 0.1 & 0.1 & 4.8 & 0.1 & 0.1 & 5.1 & 0.1 & 0.1 \\
\hline Dairy beverages & 1.0 & 1.1 & 1.0 & 0.9 & 0.8 & 0.7 & 1.1 & 1.4 & 1.2 \\
\hline Dairy desserts & 1.1 & 1.8 & 1.5 & 1.1 & 1.5 & 1.3 & 1.2 & 2.0 & 1.7 \\
\hline Yogurt & 5.5 & 6.7 & 5.8 & 5.3 & 6.8 & 6.0 & 5.6 & 6.6 & 5.5 \\
\hline Cottage cheese, quark & 0.4 & 0.4 & 0.3 & 0.3 & 0.3 & 0.2 & 0.5 & 0.5 & 0.4 \\
\hline $\begin{array}{l}\text { Bread, bread products, and } \\
\text { dough }\end{array}$ & 2.8 & 2.0 & 1.6 & 2.9 & 1.3 & 1.1 & 2.7 & 2.6 & 2.2 \\
\hline Breakfast cereals & 1.1 & 2.7 & 2.2 & 1.2 & 2.5 & 2.1 & 1.1 & 2.9 & 2.4 \\
\hline $\begin{array}{l}\text { Other starchy foods, cereals, } \\
\text { and legumes }\end{array}$ & 0.8 & 0.1 & 0.1 & 1.0 & 0.1 & 0.0 & 0.7 & 0.1 & 0.1 \\
\hline Sweet products & 28.1 & 55.4 & 47.4 & 29.2 & 54.0 & 46.5 & 27.0 & 56.7 & 48.3 \\
\hline Table sugar & 4.2 & 8.8 & 7.4 & 5.1 & 10.1 & 8.6 & 3.4 & 7.6 & 6.1 \\
\hline $\begin{array}{l}\text { Honey, jam, and other sweet } \\
\text { sauces/spreads }\end{array}$ & 8.5 & 16.6 & 14.7 & 9.4 & 17.3 & 15.2 & 7.6 & 15.9 & 14.1 \\
\hline Confectionary and candies & 3.7 & 7.3 & 6.2 & 3.2 & 5.9 & 4.9 & 4.1 & 8.7 & 7.4 \\
\hline Pure chocolate & 2.3 & 5.0 & 4.1 & 2.1 & 3.9 & 3.3 & 2.5 & 6.0 & 4.9 \\
\hline Syrups & 1.0 & 1.8 & 1.6 & 1.0 & 1.7 & 1.5 & 1.1 & 1.9 & 1.7 \\
\hline Ice cream & 1.2 & 2.5 & 2.1 & 1.2 & 2.3 & 1.9 & 1.3 & 2.6 & 2.3 \\
\hline Cakes and cookies & 7.1 & 13.4 & 11.3 & 7.2 & 12.8 & 10.9 & 7.0 & 14.0 & 11.7 \\
\hline Beverages & 19.9 & 17.4 & 29.4 & 22.8 & 20.5 & 32.4 & 17.1 & 14.2 & 26.4 \\
\hline Fruit and vegetable juices & 8.3 & 1.8 & 12.9 & 8.3 & 1.8 & 12.4 & 8.2 & 1.8 & 13.5 \\
\hline Soft drinks & 9.1 & 13.2 & 12.1 & 11.8 & 16.6 & 15.3 & 6.4 & 9.9 & 8.9 \\
\hline Water & 0.1 & 0.2 & 0.2 & 0.1 & 0.2 & 0.2 & 0.1 & 0.3 & 0.2 \\
\hline Coffee and tea & 0.8 & 0.9 & 0.8 & 0.7 & 0.8 & 0.7 & 0.9 & 1.0 & 0.8 \\
\hline Alcoholic drinks & 1.6 & 1.2 & 3.4 & 1.8 & 1.1 & 3.9 & 1.4 & 1.3 & 2.9 \\
\hline Processed meat & 0.4 & 2.1 & 1.4 & 0.5 & 2.5 & 1.5 & 0.3 & 1.7 & 1.2 \\
\hline Processed fish and seafood & 0.0 & 0.1 & 0.1 & 0.0 & 0.2 & 0.2 & 0.0 & 0.1 & 0.0 \\
\hline $\begin{array}{l}\text { Sauces, condiments, and } \\
\text { flavorings }\end{array}$ & 2.7 & 6.1 & 4.6 & 2.9 & 5.6 & 4.3 & 2.6 & 6.6 & 4.9 \\
\hline Soups & 1.1 & 0.7 & 0.5 & 0.9 & 0.6 & 0.5 & 1.3 & 0.8 & 0.6 \\
\hline Salty snacks & 0.3 & 0.5 & 0.4 & 0.3 & 0.4 & 0.3 & 0.3 & 0.6 & 0.4 \\
\hline Others/miscellaneous & 1.5 & 1.1 & 1.3 & 1.5 & 1.0 & 1.1 & 1.5 & 1.1 & 1.5 \\
\hline
\end{tabular}


Table 3. Mean contribution by food group for total, added, and free sugars by age group.

\begin{tabular}{|c|c|c|c|c|c|c|c|c|c|}
\hline \multirow[b]{2}{*}{ Food group } & \multicolumn{3}{|c|}{18 to 29 years old $(n=400$, Weighted $n=870,489)$} & \multicolumn{3}{|c|}{30 to 64 years old ( $n=1319$, Weighted $n=3,108,362$ ) } & \multicolumn{3}{|c|}{65 to 75 years old $(n=338$, Weighted $n=649,026$} \\
\hline & $\begin{array}{c}\text { Total Sugars } \\
(\%)\end{array}$ & $\begin{array}{c}\text { Added } \\
\text { Sugars (\%) }\end{array}$ & $\begin{array}{c}\text { Free Sugars } \\
(\%)\end{array}$ & $\begin{array}{c}\text { Total Sugars } \\
\mathbf{( \% )}\end{array}$ & $\begin{array}{c}\text { Added Sugars } \\
(\%)\end{array}$ & Free Sugars $(\%)$ & $\begin{array}{c}\text { Total Sugars } \\
(\%)\end{array}$ & $\begin{array}{c}\text { Added } \\
\text { Sugars (\%) }\end{array}$ & $\begin{array}{c}\text { Free Sugars } \\
(\%)\end{array}$ \\
\hline Tubercles and potato products & 0.7 & 0.5 & 0.3 & 0.5 & 0.3 & 0.3 & 0.5 & 0.4 & 0.3 \\
\hline Vegetables & 6.1 & 1.2 & 1.0 & 6.8 & 0.8 & 0.6 & 6.6 & 0.4 & 0.2 \\
\hline Fruit & 15.3 & 0.2 & 0.8 & 20.7 & 0.2 & 1.3 & 27.7 & 0.3 & 1.8 \\
\hline Nuts, seeds, and olives & 0.3 & 0.0 & 0.0 & 0.3 & 0.0 & 0.0 & 0.2 & 0.0 & 0.0 \\
\hline Dairy products & 12.6 & 8.7 & 7.5 & 13.1 & 10.2 & 8.7 & 12.8 & 10.9 & 9.4 \\
\hline Milk & 5.6 & 0.2 & 0.1 & 4.9 & 0.0 & 0.0 & 4.5 & 0.0 & 0.0 \\
\hline Dairy beverages & 1.2 & 0.8 & 0.8 & 1.0 & 1.3 & 1.1 & 0.6 & 0.6 & 0.5 \\
\hline Dairy desserts & 1.0 & 1.4 & 1.1 & 1.2 & 1.8 & 1.6 & 1.2 & 1.9 & 1.6 \\
\hline Yogurt & 4.3 & 5.8 & 5.1 & 5.6 & 6.7 & 5.7 & 6.2 & 8.0 & 7.0 \\
\hline Cottage cheese, quark & 0.6 & 0.5 & 0.3 & 0.4 & 0.4 & 0.3 & 0.4 & 0.3 & 0.3 \\
\hline $\begin{array}{l}\text { Bread, bread products, and } \\
\text { dough }\end{array}$ & 2.9 & 1.6 & 1.2 & 2.9 & 2.0 & 1.7 & 2.4 & 2.4 & 1.8 \\
\hline Breakfast cereals & 2.0 & 4.4 & 3.6 & 1.0 & 2.5 & 2.0 & 0.7 & 1.5 & 1.4 \\
\hline $\begin{array}{l}\text { Other starchy foods, cereals, } \\
\text { and legumes }\end{array}$ & 1.2 & 0.1 & 0.1 & 0.7 & 0.1 & 0.1 & 0.7 & 0.1 & 0.1 \\
\hline Sweet products & 24.2 & 43.8 & 37.6 & 28.6 & 56.2 & 48.0 & 30.8 & 67.1 & 57.8 \\
\hline Table sugar & 3.2 & 6.1 & 5.2 & 4.6 & 9.6 & 8.1 & 3.7 & 8.6 & 7.1 \\
\hline $\begin{array}{l}\text { Honey, jam, and other sweet } \\
\text { sauces/spreads }\end{array}$ & 6.4 & 11.8 & 10.4 & 8.0 & 15.5 & 13.5 & 13.4 & 28.4 & 25.8 \\
\hline Confectionary and candies & 4.9 & 9.0 & 7.8 & 3.7 & 7.3 & 6.2 & 2.2 & 5.2 & 4.3 \\
\hline Pure chocolate & 1.8 & 3.5 & 3.0 & 2.5 & 5.5 & 4.5 & 1.7 & 4.3 & 3.5 \\
\hline Syrups & 1.0 & 1.6 & 1.4 & 1.1 & 1.8 & 1.6 & 1.0 & 1.7 & 1.6 \\
\hline Ice cream & 1.2 & 2.1 & 1.8 & 1.2 & 2.3 & 2.0 & 1.5 & 3.7 & 3.2 \\
\hline Cakes and cookies & 5.7 & 9.6 & 8.0 & 7.5 & 14.1 & 12.0 & 7.3 & 15.4 & 12.4 \\
\hline Beverages & 28.0 & 27.4 & 37.7 & 19.3 & 16.6 & 28.8 & 12.5 & 7.5 & 21.0 \\
\hline Fruit and vegetable juices & 9.3 & 2.6 & 13.4 & 8.1 & 1.7 & 12.8 & 7.6 & 1.3 & 13.1 \\
\hline Soft drinks & 15.9 & 22.1 & 20.1 & 8.5 & 12.5 & 11.4 & 3.0 & 4.8 & 4.3 \\
\hline Water & 0.1 & 0.2 & 0.2 & 0.1 & 0.3 & 0.2 & 0.0 & 0.1 & 0.1 \\
\hline Coffee and tea & 0.9 & 1.1 & 1.0 & 0.9 & 0.9 & 0.8 & 0.5 & 0.5 & 0.3 \\
\hline Alcoholic drinks & 1.8 & 1.3 & 3.0 & 1.6 & 1.2 & 3.6 & 1.4 & 0.9 & 3.2 \\
\hline Processed meat & 0.5 & 2.2 & 1.8 & 0.4 & 2.1 & 1.3 & 0.4 & 1.8 & 1.1 \\
\hline Processed fish and seafood & 0.0 & 0.2 & 0.2 & 0.0 & 0.1 & 0.1 & 0.0 & 0.0 & 0.0 \\
\hline $\begin{array}{l}\text { Sauces, condiments, and } \\
\text { flavorings }\end{array}$ & 3.4 & 6.8 & 5.6 & 2.8 & 6.1 & 4.6 & 1.8 & 5.3 & 3.4 \\
\hline Soups & 0.9 & 0.8 & 0.5 & 1.1 & 0.8 & 0.6 & 1.2 & 0.5 & 0.3 \\
\hline Salty snacks & 0.4 & 0.5 & 0.3 & 0.3 & 0.5 & 0.3 & 0.3 & 0.8 & 0.5 \\
\hline Others/miscellaneous & 1.5 & 1.6 & 1.8 & 1.5 & 1.0 & 1.2 & 1.5 & 0.8 & 0.8 \\
\hline
\end{tabular}


Table 4. Adherence to international and national guidelines.

\begin{tabular}{|c|c|c|c|c|c|c|c|c|}
\hline & $n(\%)$ & Weighted $n(\%)$ & $\begin{array}{c}\text { Free Sugar: }<10 \% \text { of } \\
\text { TEI }(\%)^{2,3}\end{array}$ & $p$-Value ${ }^{4}$ & $\begin{array}{c}\text { Free Sugar: }<5 \% \text { of } \\
\text { TEI }(\%)^{5}\end{array}$ & $p$-Value ${ }^{4}$ & $\begin{array}{c}\text { Free Sugar: } \\
\text { Max. } 50 \text { g/day }{ }^{3}\end{array}$ & $p$-Value ${ }^{4}$ \\
\hline All & $2057(100)$ & $4,627,878(100)$ & $44 \%$ & & $8 \%$ & & $42 \%$ & \\
\hline $\begin{array}{l}\text { Sex } \\
\text { Men }\end{array}$ & $933(45)$ & $2,305,141(50)$ & $45 \%$ & & $9 \%$ & & $35 \%$ & \\
\hline Women & $1124(55)$ & $2,322,737(50)$ & $44 \%$ & 0.65 & $8 \%$ & 0.32 & $50 \%$ & $<0.001$ \\
\hline $\begin{array}{l}\text { Age } \\
18-29 \text { y }\end{array}$ & $400(19)$ & $870,489(19)$ & $36 \%$ & & $7 \%$ & & $34 \%$ & \\
\hline $\begin{array}{l}30-64 \text { y } \\
65-75 \text { y }\end{array}$ & $\begin{array}{l}1319(64) \\
338(16)\end{array}$ & $\begin{array}{c}3,108,362(67) \\
649,026(14)\end{array}$ & $\begin{array}{l}45 \% \\
53 \%\end{array}$ & $<0.001$ & $\begin{array}{c}8 \% \\
11 \%\end{array}$ & 0.48 & $\begin{array}{l}42 \% \\
54 \%\end{array}$ & $<0.001$ \\
\hline $\begin{array}{l}\text { Linguistic region } \\
\text { German-speaking }\end{array}$ & $1341(65)$ & $3,183,216(69)$ & $44 \%$ & & $9 \%$ & & $40 \%$ & \\
\hline $\begin{array}{l}\text { French-speaking } \\
\text { Italian-speaking }\end{array}$ & $\begin{array}{l}502(24) \\
214(10)\end{array}$ & $\begin{array}{c}1,187,738(26) \\
256,925(6)\end{array}$ & $\begin{array}{l}42 \% \\
62 \%\end{array}$ & 0.002 & $\begin{array}{c}6 \% \\
13 \%\end{array}$ & 0.039 & $\begin{array}{l}42 \% \\
64 \%\end{array}$ & $<0,001$ \\
\hline $\begin{array}{l}\text { Education (highest } \\
\text { degree) }^{1} \\
\text { No university degree }\end{array}$ & $1057(51)$ & 2,210,585 (48) & $43 \%$ & & $8 \%$ & & $43 \%$ & \\
\hline $\begin{array}{l}\text { University degree } \\
\text { Nationality }\end{array}$ & $997(48)$ & $2,405,018(52)$ & $46 \%$ & 0.44 & $9 \%$ & 0.97 & $41 \%$ & 0.46 \\
\hline Non-Swiss & $\begin{array}{l}265 \\
(13)\end{array}$ & $1,145,199(25)$ & $50 \%$ & 0.09 & $10 \%$ & 0.24 & $43 \%$ & 0.68 \\
\hline
\end{tabular}

${ }^{1}$ Three survey participants did not answer this question. ${ }^{2}$ Strong recommendation from World Health Organization. ${ }^{3}$ National recommendation from the Federal Commission on Nutrition. ${ }^{4}$ Differences between groups were estimated using chi-squared tests for weighted data. ${ }^{5}$ Conditional recommendation from World Health Organization. 


\section{Discussion}

In this representative sample of the Swiss population, total sugar intake represented $19 \%$ of TEI (mean: $107 \mathrm{~g} /$ day \pm 44 ), while added and free sugar intakes represented $9 \%$ of TEI (mean: $53 \mathrm{~g} /$ day \pm 36 ) and $11 \%$ of TEI $(65 \mathrm{~g} /$ day \pm 40$)$, respectively. Adherence to the WHO recommendations for free sugar intake was generally low, with more than half of the population $(56 \%)$ reporting a free sugar intake above the recommended $10 \%$ of TEI, and a large majority ( $92 \%$ ) consuming more than $5 \%$ of TEI from free sugar. The consumption of total, added, and free sugar was higher among young adults (18-29 years) compared to older adults (65-75 years), whereas individuals living in the Italian-speaking region had a lower mean intake of free sugar compared to the other two linguistic regions (German and French). In Swiss adults, the foods that mostly contributed to free sugar intake were sweet products, non-alcoholic beverages, and dairy products, with SSB contributing the most to free sugar intake amongst young adults.

The current consumption of total, added, and free sugars (respectively 19\%, $9 \%$, and $11 \%$ of TEI) among Swiss adults is slightly lower than the intakes reported in the Dutch adult population (19 to 69 years old), which were respectively $21 \%, 12 \%$, and $14 \%$ of TEI [38]. A recent review of nationally representative dietary surveys across the world reported that the total sugar intake among adults aged 18 to 65 ranged from $13.5 \%$ to $24.6 \%$, with Italian men having the lowest consumption and young American women having the highest [21]. Similarly, the consumption of total sugar in our survey was the lowest among individuals living in the Italian-speaking region of Switzerland. The consumption of added sugar by the Swiss population is consistent with global estimates reported by Newens et al. (2016) showing that added sugar intake ranged from $7.2 \%$ in Brazil and Norway to $16.3 \%$ in the United States (USA) [21]. Since this survey was the first of its kind in Switzerland, comparisons of the consumption of total, added, and free sugars over time could not be performed. However, worldwide trends in dietary sugar intake in 10 European countries, as well as Australia, New Zealand, and the USA suggest that dietary sugar intake values have been mainly decreasing or stable between 1971-2012, although estimates of mean population dietary sugars intake may be increasing in some specific subpopulations [41].

Consistent with our findings, a recent review of dietary sources of sugars in Europe reported that sweet products, non-alcoholic and alcoholic beverages and dairy products were the foods contributing the most to added sugar intake in both adults and children [20]. In France, sweet products accounted for $60 \%$ of total added sugar in women and $61 \%$ in men aged 18 to 79 years, whereas they respectively accounted for $53 \%$ and $47 \%$ in women and men aged 19 to 69 years living in the Netherlands (Switzerland: 57\% and 54\%) [20]. As for non-alcoholic and alcoholic beverages, they represented 12\% of the total added sugar in the diets of French women, while the comparative figures were $16 \%$ for French men, 24\% for Dutch women, and 31\% for Dutch men (Switzerland: 14\% and 21\%) [20]. The same European review [20] found that SSB alone (excluding fruit juices) accounted for $7 \%$ and $10 \%$ of total added sugar among French females and males, and $18 \%$ and $26 \%$ among Dutch females and males, respectively (Switzerland: 10\% and 17\%). Similarly, SSB and sweet bakery products were the primary contributor to added sugar intake in the USA, followed by candy and other desserts (e.g., ice cream) [22].

A few previous studies have investigated adherence to the free sugar guidelines of the WHO among adults and children [38,42]. The study in adults found that $4 \%$ of Dutch men and women aged 19 to 69 reported a free sugar consumption below $5 \%$ of TEI, while a third of the women and the men ( $29 \%$ and $33 \%$ ) observed $<10 \%$ of TEI [38]. This suggests that the adherence to the WHO guidelines was slightly better in Switzerland, despite the general relative low adherence. These differences might also be partly explained by a lower participation rate in menuCH, increasing the risk of participants being less representative of the general population, i.e., more health-conscious. In addition, although the methods used to estimate sugar consumption in the Netherlands and in Switzerland were similar (i.e., two 24HDR, modeling of usual intake, and nutrition experts to assign added sugar content in foods), we cannot exclude discrepancies. 
Two main limitations of the present study are (1) the frequent under-reporting of food items that are high in sugar, and (2) the imprecise estimations of the total, added, and free sugar contents of some food products due to constant changes in the content and ingredient quantity used by manufacturers (food reformulation), and the lack of consensus on added and free sugar definitions. To attenuate this issue, sugar content estimations were performed by two dietitians independently based on standard recipes, information on packaging, or manufacturer websites, and the decisions made were documented in a flow chart, following the example of Sluik et al. in the Netherlands [38]. Since added sugars are more narrowly defined than free sugars and because foods rich in added sugars are the main under-reported food items by both adults and children [21], the percentage of total energy intake coming from added and free sugars is likely to be even higher. Another limitation of this study was that sugar intake among children could not be estimated, since data on Swiss children's diet have not yet been collected: therefore, the present findings can only be generalized to the Swiss adult population. The main strength of this study lies in the inclusion of two non-consecutive 24HDR conducted by trained dietitians, which enabled the estimation of habitual dietary intake and the removal of some intra-individual variation using the MSM. This study is also the first ever to report data regarding sugar consumption in a relatively representative sample of the Swiss adult population.

\section{Conclusions}

In conclusion, more than half of women and men had free sugar intakes above the updated WHO recommendation of $10 \%$ of TEI, and $92 \%$ of the population had an intake of free sugar above $5 \%$ of TEI. Future surveys should include data about children's dietary intake, but available data among adults show that those within the Swiss population consume more sugar than they should, and a major public health effort would be necessary to increase the adherence to the recommendations.

Supplementary Materials: The following are available online at http://www.mdpi.com/2072-6643/11/5/1117/s1, Figure S1: Overview of the different types of carbohydrates. Figure S2: Overview of the estimation of free and added sugars in all consumed foods and beverages, Table S1: Decision flow chart for the estimated percentage of added and free sugars out of total sugars.

Author Contributions: A.K. wrote the study protocol and retrieved the data. A.C. and P.G. assigned values for total (when missing), added, and free sugar to all consumed foods reported by survey participants. A.C. conducted the statistical analyses. A.K. and A.C. contributed to the concept and the design of the manuscript and wrote it. All the authors have revised the protocol and participated in the writing of the manuscript. All the authors approved the final manuscript. None of the authors report any conflict of interest related to the study.

Funding: The National Nutrition Survey menuCH was co-funded by the Swiss Federal Food Safety and Veterinary Office and the Federal Office of Public Health. The work related to this project received no funding.

Acknowledgments: The authors express their gratitude to all survey participants. Additionally, we thank the Swiss Federal Food Safety and Veterinary Office, www.blv.admin.ch, for providing data from the Swiss National Nutrition Survey menuCH 2014-2015. In addition, the authors would like to acknowledge the contribution of Jo Martin Jewel from the World Health Organization Regional Office for Europe, Copenhagen Denmark-Nutrition, Physical Activity and Obesity Programme-Division of Noncommunicable Diseases and Promoting Health through the Life-Course, who provided feedback and participated in the writing of the protocol.

Conflicts of Interest: The authors declare no conflict of interest. The survey funders, the Federal Food Safety and Veterinary Office, assigned the nutrient values (total sugars) to foods reported by survey participants using the Swiss Food Composition Database (linkage). They provided free access to data. They had no direct role in study design, data analyses, interpretation of results, manuscript writing, or decision to publish the results.

\section{References}

1. Te Morenga, L.; Mallard, S.; Mann, J. Dietary sugars and body weight: Systematic review and meta-analyses of randomised controlled trials and cohort studies. BMJ 2012, 346, e7492. [CrossRef]

2. Keller, A.; Torre, S.B. Della sugar-sweetened beverages and obesity among children and adolescents: A review of systematic literature reviews. Child. Obes. 2015, 11. [CrossRef]

3. Malik, V.S.; Pan, A.; Willett, W.C.; Hu, F.B. Sugar-sweetened beverages and weight gain in children and adults: A systematic review and meta-analysis. Am. J. Clin. Nutr. 2013, 98, 1084-1102. [CrossRef] [PubMed] 
4. Moynihan, P. Sugars and dental caries: Evidence for setting a recommended threshold for intake. Adv. Nutr. 2016, 7, 149-156. [CrossRef]

5. Moynihan, P.J.; Kelly, S.A.M. Effect on caries of restricting sugars intake. J. Dent. Res. 2014, 93, 8-18. [CrossRef] [PubMed]

6. de Koning, L.; Malik, V.S.; Rimm, E.B.; Willett, W.C.; Hu, F.B. Sugar-sweetened and artificially sweetened beverage consumption and risk of type 2 diabetes in men. Am. J. Clin. Nutr. 2011, 93, 1321-1327. [CrossRef]

7. Malik, V.S.; Popkin, B.M.; Bray, G.A.; Despres, J.-P.; Willett, W.C.; Hu, F.B. Sugar-sweetened beverages and risk of metabolic syndrome and type 2 diabetes: A meta-analysis. Diabetes Care 2010, 33, 2477-2483. [CrossRef]

8. Yu, Z.; Ley, S.H.; Sun, Q.; Hu, F.B.; Malik, V.S. Cross-sectional association between sugar-sweetened beverage intake and cardiometabolic biomarkers in US women. Br. J. Nutr. 2018, 119, 570-580. [CrossRef] [PubMed]

9. Keller, A.; Heitmann, B.L.; Olsen, N. Sugar-sweetened beverages, vascular risk factors and events: A systematic literature review. Public Health Nutr. 2014, 18, 1-10. [CrossRef] [PubMed]

10. Fuchs, M.A.; Sato, K.; Niedzwiecki, D.; Ye, X.; Saltz, L.B.; Mayer, R.J.; Mowat, R.B.; Whittom, R.; Hantel, A.; Benson, A.; et al. Sugar-sweetened beverage intake and cancer recurrence and survival in CALGB 89803 (Alliance). PLoS ONE 2014, 9, e99816. [CrossRef] [PubMed]

11. Van Blarigan, E.L.; Meyerhardt, J.A. Role of physical activity and diet after colorectal cancer diagnosis. J. Clin. Oncol. 2015, 33, 1825-1834. [CrossRef] [PubMed]

12. Port, A.M.; Ruth, M.R.; Istfan, N.W. Fructose consumption and cancer. Curr. Opin. Endocrinol. Diabetes Obes. 2012, 19, 367-374. [CrossRef] [PubMed]

13. Wijarnpreecha, K.; Thongprayoon, C.; Edmonds, P.J.; Cheungpasitporn, W. Associations of sugar-and artificially sweetened soda with nonalcoholic fatty liver disease: A systematic review and meta-analysis. QJM 2016, 109, 461-466. [CrossRef]

14. An, Y.; Varma, V.R.; Varma, S.; Casanova, R.; Dammer, E.; Pletnikova, O.; Chia, C.W.; Egan, J.M.; Ferrucci, L.; Troncoso, J.; et al. Evidence for brain glucose dysregulation in Alzheimer's disease. Alzheimer's Dement. 2018, 14, 318-329. [CrossRef] [PubMed]

15. Pase, M.P.; Himali, J.J.; Jacques, P.F.; DeCarli, C.; Satizabal, C.L.; Aparicio, H.; Vasan, R.S.; Beiser, A.S.; Seshadri, S. Sugary beverage intake and preclinical Alzheimer's disease in the community. Alzheimer's Dement. 2017, 13, 955-964. [CrossRef]

16. WHO. Guideline: Sugars intake for adults and children. Available online: https://apps.who.int/iris/bitstream/ handle/10665/149782/9789241549028_eng.pdf;jsessionid=EFC3B0F9BB09F52DD42BF59E35690A81? sequence $=1$ (accessed on 17 May 2019).

17. Health at a Glance: Europe 2018. OECD, 2018. Available online: https://www.oecd.org/health/health-at-aglance-europe-23056088.htm (accessed on 17 May 2019).

18. Obesity Update-OECD. Available online: http://www.oecd.org/health/obesity-update.htm (accessed on 26 March 2019).

19. Federal Commission for Nutrition. Recommendations of the Federal Commission for Nutrition on Carbohydrate Intake. Available online: https://www.eek.admin.ch/eek/en/home/pub/kohlenhydrate-in-derernaehrung-.html (accessed on 17 May 2019).

20. Azaïs-Braesco, V.; Sluik, D.; Maillot, M.; Kok, F.; Moreno, L.A. A review of total \& added sugar intakes and dietary sources in Europe. Nutr. J. 2017, 16, 6. [CrossRef]

21. Newens, K.J.; Walton, J. A review of sugar consumption from nationally representative dietary surveys across the world. J. Hum. Nutr. Diet 2016, 29, 225-240. [CrossRef]

22. Bailey, R.; Fulgoni, V.; Cowan, A.; Gaine, P. Sources of added sugars in young children, adolescents, and adults with low and high intakes of added sugars. Nutrients 2018, 10, 102. [CrossRef]

23. Nutri-Score Nutritional Score and Color grades. Available online: https://world.openfoodfacts.org/nutriscore (accessed on 22 March 2019).

24. Yeung, C.H.C.; Gohil, P.; Rangan, A.M.; Flood, V.M.; Arcot, J.; Gill, T.P.; Louie, J.C.Y. Modelling of the impact of universal added sugar reduction through food reformulation. Sci. Rep. 2017, 7, 17392. [CrossRef]

25. Iacobucci, G. Government urges food industry to cut $20 \%$ of sugar and reduce portion sizes. BMJ 2016, 355, i5348. [CrossRef]

26. Powell, L.M.; Maciejewski, M.L. Taxes and Sugar-Sweetened Beverages. JAMA 2018, 319, 229-230. [CrossRef] [PubMed] 
27. Swiss Nutrition Strategy. Available online: https:/www.blv.admin.ch/blv/en/home/das-blv/strategien/ schweizer-ernaehrungsstrategie.html (accessed on 4 March 2019).

28. Chatelan, A.; Beer-Borst, S.; Randriamiharisoa, A.; Pasquier, J.; Blanco, J.; Siegenthaler, S.; Paccaud, F.; Slimani, N.; Nicolas, G.; Camenzind-Frey, E.; et al. Major differences in diet across three linguistic regions of Switzerland: Results from the First National Nutrition Survey menuCH. Nutrients 2017, 9, 1163. [CrossRef] [PubMed]

29. Federal Statistical Office Stichprobenrahmen für Personen- und Haushaltserhebungen (Swiss persons and households registry). Available online: https://www.bfs.admin.ch/bfs/de/home/grundlagen/volkszaehlung/ volkszaehlung-teil-gesamtsystem/stichprobenrahmen.html (accessed on 7 May 2019).

30. Slimani, N.; Casagrande, C.; Nicolas, G.; Freisling, H.; Huybrechts, I.; Ocke, M.C.; Niekerk, E.M.; van Rossum, C.; Bellemans, M.; De Maeyer, M.; et al. The standardized computerized 24-h dietary recall method EPIC-Soft adapted for pan-European dietary monitoring. Eur J. Clin. Nutr. 2011, 65, S5-S15. [CrossRef]

31. Crispim, S.P.; de Vries, J.H.; Geelen, A.; Souverein, O.W.; Hulshof, P.J.; Lafay, L.; Rousseau, A.S.; Lillegaard, I.T.; Andersen, L.F.; Huybrechts, I.; et al. Two non-consecutive $24 \mathrm{~h}$ recalls using EPIC-Soft software are sufficiently valid for comparing protein and potassium intake between five European centres-results from the European Food Consumption Validation (EFCOVAL) study. Br. J. Nutr. 2011, 105, 447-458. [CrossRef]

32. Camenzind-Frey, E.; Zuberbuehler, C.A. menuCH-Schweizerisches Fotobuch/Livre Photo Suisse/Manuale Fotografico Svizzero (mеnuCH picture book); Federal Office of Public Health \& Federal Food Safety and Veterinary Office: Bern, Switzerland, 2014.

33. Federal Food Safety and Veterinary Office Swiss Food Composition Database. Available online: http: //naehrwertdaten.ch (accessed on 7 May 2019).

34. Presser, K.; Weber, D.; Norrie, M. FoodCASE: A system to manage food composition, consumption and TDS data. Food Chem. 2018, 238, 166-172. [CrossRef]

35. Mela, D.J.; Woolner, E.M. Perspective: Total, added, or free? What kind of sugars should we be talking about? Adv. Nutr. 2018, 9, 63-69. [CrossRef] [PubMed]

36. French Agency for Food Environmental and Occupational Health \& Safety (ANSES) Ciqual-Table de composition nutritionnelle des aliments (French Food Composition Table). Available online: https:/ciqual. anses.fr/ (accessed on 7 May 2019).

37. Bowman, S.A. Added sugars: Definition and estimation in the USDA Food Patterns Equivalents Databases. J. Food Compos. Anal. 2017, 64, 64-67. [CrossRef]

38. Sluik, D.; van Lee, L.; Engelen, A.; Feskens, E. Total, Free, and Added Sugar Consumption and Adherence to Guidelines: The Dutch National Food Consumption Survey 2007-2010. Nutrients 2016, 8, 70. [CrossRef] [PubMed]

39. Swan, G.E.; Powell, N.A.; Knowles, B.L.; Bush, M.T.; Levy, L.B. A definition of free sugars for the UK. Public Heal. Nutr 2018, 21, 1636-1638. [CrossRef]

40. Harttig, U.; Haubrock, J.; Knuppel, S.; Boeing, H.; Consortium, E. The MSM program: Web-based statistics package for estimating usual dietary intake using the Multiple Source Method. Eur. J. Clin. Nutr. 2011, 65, S87-S91. [CrossRef] [PubMed]

41. Wittekind, A.; Walton, J. Worldwide trends in dietary sugars intake. Nutr. Res. Rev. 2014, $27,330-345$. [CrossRef] [PubMed]

42. Louie, J.C.Y.; Moshtaghian, H.; Rangan, A.M.; Flood, V.M.; Gill, T.P. Intake and sources of added sugars among Australian children and adolescents. Eur. J. Nutr. 2016, 55, 2347-2355. [CrossRef] [PubMed]

(C) 2019 by the authors. Licensee MDPI, Basel, Switzerland. This article is an open access article distributed under the terms and conditions of the Creative Commons Attribution (CC BY) license (http://creativecommons.org/licenses/by/4.0/). 